\title{
Discovery of Trichopria keralensis (Hymenoptera, Diaprioidea, Diapriidae) in South Korea and Japan, a review of the keralensis species group of Trichopria and the nomenclature and synonymy of Alareka
}

\author{
Chang-Jun Kim', David G. Notton², Jong-Wook Lee
}

I Division of Forest Biodiversity, Korea National Arboretum, Pocheon-si, Gyeonggi-Province, Republic of Korea 2 Department of Life Sciences, Insects Division, Darwin Centre - room 315, The Natural History Museum, Cromwell Road, London, SW7 5BD, United Kingdom 3 Department of Life Sciences, Yeungnam University, Gyeongsan-si, Gyeongsangbuk-Province, Republic of Korea

Corresponding author:Jong-Wook Lee (jwlee1@ynu.ac.kr)

Academic editor: M. Yoder | Received 18 March 2016 | Accepted 4 October 2016 | Published 28 October 2016

http://zoobank.org/947F34B1-6639-4CB2-8AEA-712C3861A9B5

Citation: Kim C-J, Notton DG, Lee J-W (2016) Discovery of Trichopria keralensis (Hymenoptera, Diaprioidea, Diapriidae) in South Korea and Japan, a review of the keralensis species group of Trichopria and the nomenclature and synonymy of Alareka. Journal of Hymenoptera Research 52: 143-151. doi: 10.3897/jhr.52.8546

\begin{abstract}
Trichopria keralensis is recorded for the first time from South Korea and Japan and a detailed redescription and photographs of this species are given. The genus Alareka is a junior synonym of Trichopria syn. $\mathbf{n}$. Alareka keralensis is transferred to Trichopria keralensis comb. n. T. keralensis and closely related species are referred to the keralensis species group of Trichopria. The keralensis species group is recorded here for the first time from the East Palaearctic, West Palaearctic, Afrotropical and Australian (Oceanian) regions. The multiple original spellings of Alareka, Alarika, Alareka keralensis and Alareka keralaensis are resolved.
\end{abstract}

\section{Keywords}

Diapriinae, Diapriini, multiple original spellings, new combination, new distribution records, synonymy

Copyright Chang-Jun Kim et al. This is an open access article distributed under the terms of the Creative Commons Attribution License (CC BY 4.0), which permits unrestricted use, distribution, and reproduction in any medium, provided the original author and source are credited. 


\section{Introduction}

Alareka, a monotypic genus of subfamily Diapriinae, was established Rajmohana and Narendran (2000) for a single Oriental species, A. keralensis, described on the basis of one male specimen collected in Kerala, India. Rajmohana and Narendran (2000) distinguished Alareka from other Diapriinae genera using a range of characters, most notably including: nasiform head; rim of frontal shelf serrated and with small median prominence; and opisthognathous mouthparts. The female of $A$. keralensis (as A. keralaensis) was recently described by Liu and Xu (2011) from Southern China. Specimens agreeing with $A$. keralensis were discovered during the present study and the known range is extended from India, China to Japan (Oriental) and South Korea (East Palaearctic) and a detailed redescription and photographs of this species are given.

Detailed examination of the new material showed that the genus Alareka is a junior synonym of Trichopria syn. $\mathrm{n}$. and consequently Alareka keralensis is transferred to Trichopria keralensis comb. $\mathrm{n}$. The synonymy is discussed in the light of the repeated and rapid evolution of a linked character suite of head characters: elongate head, opisthognathous mouthparts and projections of the frons and antennal shelf, which has arisen frequently and repeatedly in Diapriidae and other microhymenoptera; also the existence of species of Trichopria evidently closely related to T. keralensis but with some head characters intermediate between Alareka and other Trichopria.

There are a number of species which are closely related to $T$. keralensis which share the extreme nasiform condition of the head and so T. keralensis and these closely related species are referred to the keralensis species group of Trichopria. This species group is defined here. The keralensis species group is recorded here for the first time from the East Palaearctic, West Palaearctic, Afrotropical and Australian (Oceanic) regions.

Unfortunately Rajmohana and Narendran (2000) gave multiple original spellings of Alareka, Alarika, Alareka keralensis and Alareka keralaensis which are resolved below in the interests of nomenclatural consistency and stability.

\section{Materials and methods}

The terminology used in the present study follows that of Nixon (1980) and Masner and García (2002). The images were captured with an Axiocam HRc camera through a Discovery V20 stereomicroscopy (Carl Zeiss, Oberkochen, Germany) and were produced with AxioVision40AC software (Carl Zeiss, Oberkochen, Germany). Final plates were prepared in Adobe Photoshop CS6 (Adobe Systems Incorporated, San Jose, United States of America).

The following abbreviations are used throughout the text: POL, distance between the inner edges of the two lateral ocelli; OOL, distance from the outer edge of a lateral ocellus to the compound eye; MT, Malaise trap. 


\section{Taxonomy}

\section{Order Hymenoptera Family Diapriidae Haliday, 1833}

\section{Trichopria Ashmead, 1893}

Phaenopria Ashmead, 1893

Planopria Kieffer, 1906

Xyalopria Kieffer, 1907

Orthopria Kieffer, 1911

Ashmeadopria Kieffer, 1912

Rhopalopria Kieffer, 1912

Abothropria Kieffer, 1913

Scapopria Kieffer, 1913

Neodiapria Kieffer, 1916

Alareka Rajmohana \& Narendran, 2000 syn. n.; Rajmohana and Narendran 1999; 178, 180 , nomen nudum; Rajmohana and Narendran 2000a: 193, nomen nudum; Rajmohana and Narendran 2000b: multiple original spellings Alareka pp. 21, 22, 23, fig, 22 and Alarika, p. 27, of which Alareka is the correct original spelling (ICZN 1999, Art. 32.5.1); Rajmohana 2006: iv, 8, 23, 26; Liu, Chen and Xu 2011: 181; Nielsen and Buffington 2011: 613; Rajmohana and Bijoy 2012: 4 unpublished work; Rajmohana, Poorani, Shweta and Malathi 2013 unpublished work.

Remarks. Before continuing further with a review of the morphology and classification of this taxon it is first necessary to clarify the confused nomenclatural history and spelling of the name Alareka: firstly it was published as a nomen nudum twice by Rajmohana and Narendran (1999, 2000a); it was first made available by Rajmohana and Narendran (2000b) but with the multiple original spellings Alareka and Alarika - the stated etymology was that it is an anagram of Kerala, and it was given feminine gender, so it can be assumed that this includes the feminine gender ending -a, i.e. the intended spelling was Alareka and that Alarika was a lapsus calami. Alareka Rajmohana \& Narendran, 2000 is therefore available and Alarika is an incorrect original spelling (ICZN 1999, Art. 32.5.1) must be corrected, and is not available.

New material of this genus was discovered the collections of Yeungnam University and The Natural History Museum, London showing that Alareka keralensis was more widespread than previous known and prompting a review of the status of the genus. A detailed redescription was made for Alareka (see Trichopria species group keralensis Description) below, which showed that some significant features had previously been missed or misinterpreted. A number of significant characters place this genus within Trichopria, specifically close to, if not within the group of species with males having whorled hairs on the antenna. These characters are: whorled hairs on the male antenna; male a 3 and a 4 normally articulated; slender fore-tibial spine present; mesoscutal hairs 
arranged in (1-)3 pairs. Alareka is highly derived in many ways but its derived features are extreme developments of characters already noted for Trichopria, such as: foamy structures made of large hyaline scales, which are found in e.g. T. drosophilae (Perkins, 1910); strongly compressed antennal scape found in e.g. T. vulgaris (Kieffer, 1912) and $T$. drosophilae; strongly laterally compressed flagellum in both sexes found in e.g. T. drosophilae.

The most significant difference from Trichopria is the nasiform head and opisthognathous mouthparts; however there are several undescribed species of Trichopria from s. e. Asia which have subtriangular heads intermediate between Alareka and the normal subround head found in most Trichopria. Such differences in the degree of development of a nasiform head, and especially opisthognathous mouthparts, are not unusual in diapriids, even within the same genus. There is a linked character suite of head characters: elongate head, opisthognathous mouthparts and projections of the frons and antennal shelf which has arisen rapidly and repeatedly in Diapriidae and other microhymenoptera (Nielsen and Buffington 2011). There are a number of diapriid genera including both opisthognathous and hypognathous species for example: Synacra Förster, 1856, Basalys Westwood, 1833, Aneuropria Kieffer, 1905 all of which include both opisthognathous and hypognathous species, and Vadana Rajmohana \& Narendren, 2000 (hypognathous) is just a Calogalesus Kieffer, 1912 (opisthognathous). While it may be easy to recognize small groups of derived opisthognathous species, and both practical and useful to key them separately for the purposes of identification, it does not serve the purposes of classification to split these off as separate genera if it leaves larger genera paraphyletic as appears to be the case of Alareka and closely related Trichopria. In any case, other more senior generic names are already available for the group of Trichopria having males with whorled hairs, should the genus be divided in future. We conclude therefore that Alareka is just a small derived subgroup of Trichopria probably close to or from within the group of species having males with whorled hairs on the antenna as exemplified by T. verticillata (Latreille, 1805). Hence the genus Alareka is considered here to be a junior synonym of Trichopria syn. n. As a consequence of the new generic synonymy Alareka keralensis is transferred to Trichopria keralensis comb. $\mathrm{n}$. Other generic synonymy given above is based on Johnson (1992) and Notton (2004, 2014).

There are a number of undescribed species which are closely related to $T$. keralensis and so $T$. keralensis and these closely related species are referred to here as the keralensis species group of Trichopria. This species group is defined here:

\section{Trichopria species group keralensis}

Description. Head nasiform; antennal shelf strongly prominent, its anterior margin with a small median prominence, and with small subtriangular lateral projections; face with strong medial carina in upper third; clypeus narrow but prominent, convex; tentorial pits deep and close together; malar sulcus absent; compound eyes with 
long, sparse hairs. Mouthparts strongly opisthognathous, mandibles elongate, together beak-like, with tips serrated. Female antenna 12-segmented; scape strongly flattened in basal 3/5; basal flagellar segments strongly laterally compressed, particularly the base of the segments; clava gradually incrassate apically, and distinctly to weakly laterally compressed; multiporous hair sensilla present on a7-12; a12 about as long as, or very slightly longer than the subapical. Male antenna 14-segmented; scape strongly flattened in basal 3/5; flagellar segments clavate, strongly laterally compressed, with a whorl of long hairs on each node, near the apex of the flagellum the nodes become subapical to medial; fourth segment usually not or weakly modified, occasionally with apical expansion and lateral emargination; a8, or a8 and a9, each with a comb of short fine hairs. Mesoscutum smooth, weakly concave laterally; notauli and humeral sulci absent; propodeum with a strong medial keel, which is raised anteriorly; dorsal propodeal areas with serried, adpressed hairs; metapleuron with small glabrous patch anteriorly. Wings well developed; subcostal, marginal and stigmal veins present, tubular, the stigmal vein very short, pointed; wing lamina densely hairy, with a small bare patch just apical of the marginal vein. Femora strongly laterally compressed basally; slender spine present at apex of fore tibia. Petiole slightly swollen medially; large tergite basally unnotched; apex of gaster slightly upturned; tip of ovipositor exserted at rest. Body colour variously blackish-brown, reddish-brown, yellowish-brown, largely smooth and shiny. Large hyaline scales (with the appearance of foam) present on anterior pronotum, propleuron, axillae, a small patch medioventrally in front of the mid coxae, and ventrally between the mid and hind coxae, and dorsal petiole; postgenal hair cushion dense, but without large transparent scales; mesoscutum of female with usually with 3 pairs of hairs, a lateral pair (next to tegula) an anterior pair and a medial pair, the male usually with only the laterals. Body length $1.80-2.84 \mathrm{~mm}$.

Remarks. The keralensis species group is distinguished from other Trichopria by the extreme nasiform, opisthognathous form of the head and mouthparts.

Distribution. The keralensis species group is recorded here for the first time from the East Palaearctic (South Korea, Japan), West Palaearctic (Saudi Arabia, Yemen), Afrotropic (Kenya, Malawi, Nigeria, Zimbabwe) and Australian - Oceanian regions (Solomon Islands). Specimens from South Korea and Japan are identified as Trichopria keralensis and a detailed redescription is provided below.

\section{Trichopria keralensis (Rajmohana \& Narendran, 2000), comb. $\mathbf{n}$.}

Figs 1-8

\section{Alareka keralensis: Rajmohana \& Narendran, 1999; 178, nomen nudum}

Alareka keralensis Rajmohana \& Narendran, 2000b: multiple original spellings keralensis pp. 21, 23, figs 1-6 and keralaensis, p. 21, of which keralensis is the correct original spelling following first reviser action by Rajmohana, 2006 Alareka keralaensis: Rajmohana and Narendran 2000b: 21, incorrect original spelling Alareka keralensis: Rajmohana 2006: iv, 8, 25, 26 
Alareka keralaensis: Liu, Chen and Xu 2011: 181, 182, 183, incorrect subsequent spelling Alareka keralensis: Rajmohana and Bijoy 2012: 4, unpublished work

Alareka keralensis: Rajmohana, Poorani, Shweta and Malathi 2013, unpublished work

Material examined ( $4 q 96 \hat{\jmath})$. SOUTH KOREA: $1 \hat{\jmath}$, Chungcheongnam-do, Seosan-si, Daegok-ri, Hanseo Univ., 18 Jul.-14 Aug. 2006 (MT), J.W. Lee; 1§̂, Gangwon-do, Hongcheon-gun, Bukbang-myeon, Nature Environment Research Park, 354' 15.6"N, 12751'1.7"E, 21 Jun.-4 Jul. 2011 (MT), J.O. Lim; 10', ditto, 16-31 Jul. 2012 (MT), J.O. Lim; 1ㅇ, ditto, 1-8 Aug. 2011 (MT), J.O. Lim; 1어, ditto, 5-18 Aug. 2011 (MT), J.O. Lim; 1§̄, Gangwon-do, Wonju-si, Heungeop-myeon, Maeji-ri 234, Yonsei University, 28 Aug.-27 Sep. 2013 (MT), H.Y. Han; 19, Gyeongsangbukdo, Cheongdo-gun, Gakbuk-myeon, Mt. Biseulsan, 15-19 Aug. 2015 (MT), J.W.

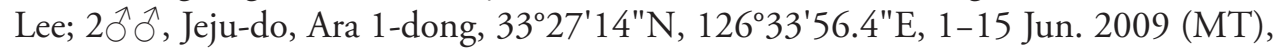
J.W. Lee. JAPAN: 19, Okinawa, Yona, Rhykyu Univ. Res. Station., May 1999, B.J. Sinclair. (all specimens housed in the collection of the Department of Life Sciences, Yeungnam University).

Redescription. Female. Head. Head smooth with long sparse hairs, in dorsal view longer than wide (19: 15), in lateral view slightly longer than high (19: 18); distinctly nasiform, antennal shelf sharply projecting, anteriorly with small medial projection, and lateral subtriangular projections; upper third of face with a median longitudinal carina, POL:OOL=3:7; eye moderately small, with sparse long hairs, distinctly shorter than postgena (3: 5); eye height much shorter than malar space (15: 22); postgenal cushion well developed; mandibles elongate, beak-like projecting, with serrated tips; antenna much shorter than body length (3: 5), gradually expanded 6-segmented clava; antennal segments a1-12 respectively in following proportions (length: width): 34: 5; 9: 4; 9: 3; 8:3; 10:3; 10:4; 9: 4; 9: 5; 9:5; 8: 6; 7:6; 10:5.

Mesosoma. Mesosoma in dorsal view equal to width of head and much shorter than long (7: 3); cervix long and smooth; pronotum and propleura with dense cushions; mesoscutum smooth, notauli, parapsidal impressions and humeral sulci completely absent; anterior scutellar pit small and deep with longitudinal carinae at bottom, much smaller than remaining scutellar disc (6: 11); scutellar disc rectangular, without lateral and posterior pits; dorsellum with 3 keels, and the median keel distinctly raised; mesosoma in lateral view clearly longer than high (10: 7); lateral part of pronotum smooth; mesopleuron smooth, with sparse hairs, without sternaulus; metapleuron with long dense hairs; median propodeal keel moderately raised, not sharply pointed, not exceeding level of mesoscutum; nucha clearly visible in dorsal view.

Wing. Fore wing with subcostal, marginal and stigmal veins tubular, stigmal vein very short, pointed.

Metasoma. Petiole covered with dense hairs, in dorsal view elongate (10: 20); second tergite (T2) without hairs and median basal notch.

Colour. Body dark brown to blackish brown; antenna yellowish brown with A8A12 dark brown; legs yellowish brown; tegulae yellowish brown, hyaline; wings hyaline with brown hairs, veins yellowish brown. 

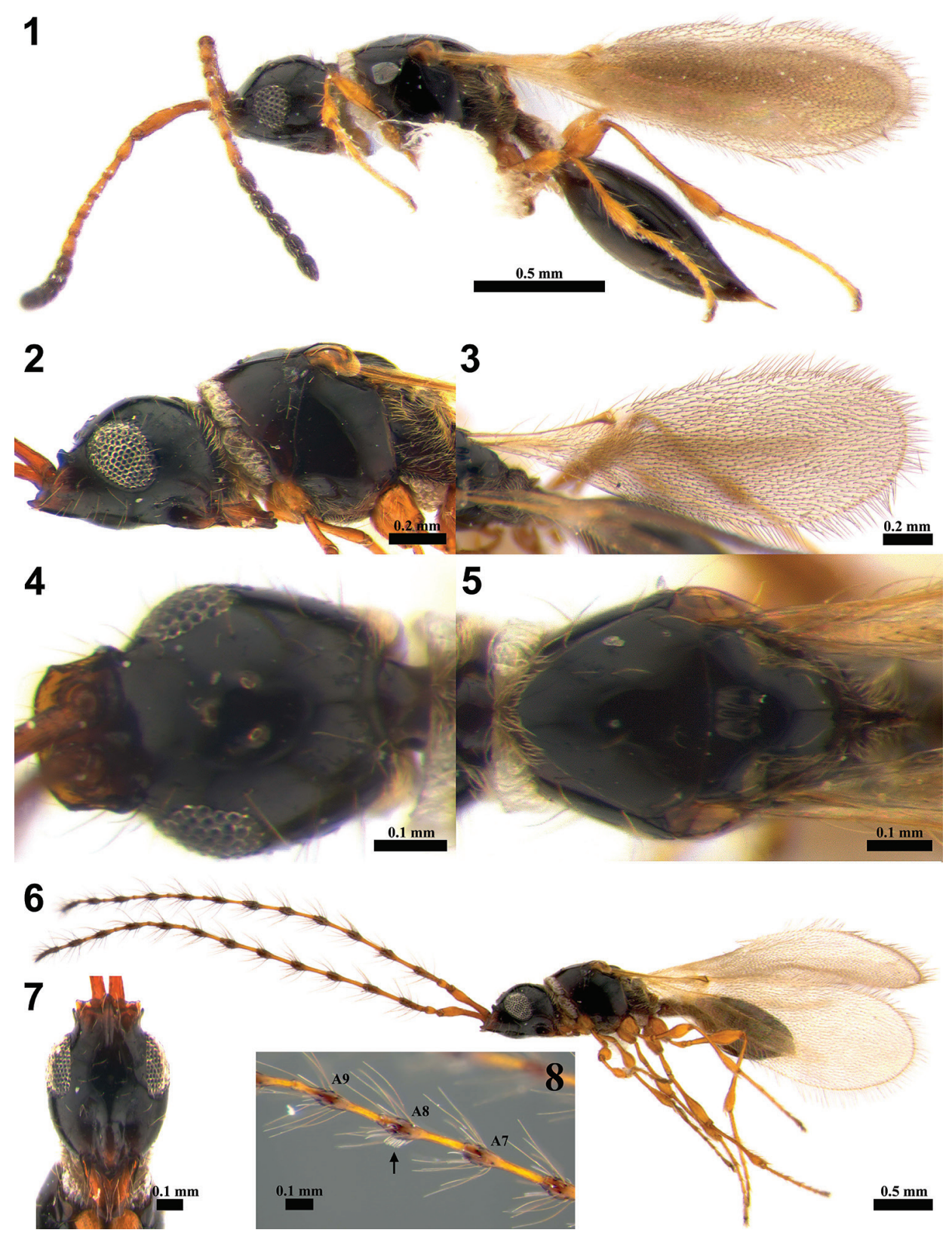

Figures I-8. Trichopria keralensis (Rajmohana \& Narendran, 2000). I Female habitus, lateral 2 Female head and mesosoma, lateral $\mathbf{3}$ Female fore wing, dorsal $\mathbf{4}$ Female head, dorsal $\mathbf{5}$ Female mesosoma, dorsal 6 Male habitus, lateral 7 Male head and face, frontal 8 Male antenna (A7-A9), lateral.

Measurements. Head length $0.43 \mathrm{~mm}$, width $0.34 \mathrm{~mm}$; mesosoma length 0.79 $\mathrm{mm}$, width $0.34 \mathrm{~mm}$; metasoma length $1.15 \mathrm{~mm}$; fore wing length $2.63 \mathrm{~mm}$; total body length $2.10-2.37 \mathrm{~mm}$. 
Male. Body length 1.80-2.84 mm. Differs from female as follows: Antenna 14-segmented, about 1.6 times the length of the body; flagellum elongate-knotted, with long hairs arranged in a whorl on each node, A8 with a comb of short fine hairs; antennal segments a1-12 respectively in following proportions (length: width): 34: 7; $8: 5 ; 18: 5 ; 26: 5 ; 29: 5 ; 28: 5 ; 26: 5 ; 29: 6 ; 29: 4 ; 26: 4 ; 25: 4 ; 24: 4 ; 23: 4 ; 30: 3$; anterior scutellar pit usually almost smooth, some males have the anterior scutellar pit almost smooth to weak or strong longitudinal carinae at bottom.

Variations. The female of the type specimen has the antenna brown with the apical four segments blackish brown, but South Korean and Japanese female specimens have antenna brown with apical five segments dark brown.

Host. Unknown.

Distribution. South Korea (new record), Japan (new record), China (Guangdong, Hainan, Yunan), India (Kerala).

Nomenclature. Just as for the genus Alareka name above the nomenclatural history of the specific epithet keralensis is confused: firstly it was published as a nomen nudum by Rajmohana and Narendran (1999); it was first made available by Rajmohana and Narendran (2000b) but with the multiple original spellings keralensis and keralaensis the stated etymology is that it was based on the collection locality Kerala, but this does not help as both spellings can be derived from this place name, keralaensis however appears to be an error since it is used only once. This unsatisfactory situation is resolved as follows; as one of the original authors, Rajmohana (2006) may be considered first reviser (ICZN 1999, Art. 24.2.4) since she used the spelling keralensis, this becomes the correct original spelling, and keralaensis becomes incorrect and unavailable (ICZN 1999, Art. 24.2.3).

\section{Acknowledgements}

The authors thank Dr. Lubomir Masner (Canadian National Collection of Insects, Ottawa, Canada) for kindly reading and providing comments on the manuscripts. This work was supported by a grant from the National Institute of Biological Resources (NIBR), funded by the Ministry of Environment (MOE) of the Republic of Korea (NIBR201601203 and NIBR201601207).

\section{References}

Haliday AH (1833) An essay on the classification of the parasitic Hymenoptera of Britain which correspond with the Ichneumones minuti of Linnaeus. Entomological Magazine 1(3): 259-276.

International Commission on Zoological Nomenclature (1999) International Code of Zoological Nomenclature, $4^{\text {th }}$ edition, International Trust for Zoological Nomenclature, The Natural History Museum, London, 306 pp. 
Johnson NF (1992) Catalog of World species of Proctotrupoidea, exclusive of Platygastridae (Hymenoptera). Memoirs of the American Entomological Institute 51: 1-825.

Liu J, Chen H, Xu Z (2011) Description of female of Alareka keralaensis Rajmohana \& Narendran (Hymenoptera: Diapriidae) from China. Oriental Insects 45(2-3): 181-185. doi: 10.1080/00305316.2011.646828

Masner L, García Rodríguez JL (2002) The genera of Diapriinae (Hymenoptera: Diapriidae) in the New World. Bulletin of the American Museum of Natural History 268: 1-138. doi: 10.1206/0003-0090(2002)268<0001:TGODHD>2.0.CO;2

Nielsen M, Buffington ML (2011) Redescription of Stentorceps Quinlan, 1984 (Hymenoptera: Figitidae), with a description of five new species. African Entomology 19(3): 597-613. doi: 10.4001/003.019.0305

Nixon GEL (1980) Diapriidae (Diapriinae) Hymenoptera, Proctotrupoidea. Handbooks for the Identification of British Insects 8(3di): 1-55.

Notton DG (2004) A catalogue of types of Diapriinae (Hymenoptera, Diapriidae) at the National Museum of Natural History, Paris, with notes on the classification of Diapriinae and a brief history of the types of Jean-Jacques Kieffer (1856-1925). Zoosystema 26(2): $315-352$.

Notton DG (2014) A catalogue of the types of Diapriinae (Hymenoptera, Diapriidae) at the Natural History Museum, London. European Journal of Taxonomy 75: 1-123. doi: $10.5852 /$ ejt. 2014.75

Rajmohana K (2006) Studies on Proctotrupoidea and Platygastroidea (Hymenoptera: Insecta) of Kerala. Memoirs of the Zoological Survey of India 21: 1-153.

Rajmohana K, Bijoy C (2012) Checklist of Diapriidae and Proctotrupidae (Hymenoptera: Insecta) of India [Online]. Zoological Survey of India. http://zsi.gov.in/checklist/Diapriidae\%20 and\%20Proctotrupidae_Hymenoptera\%20Insecta.pdf [accessed 14 Jan. 2013]

Rajmohana K, Narendran TC (1999) Species diversity in diapriine genera (Hymenoptera: Proctotrupoidea) of India. Entomon 24: 177-183.

Rajmohana K, Narendran TC (2000a) Descriptions of a new genus Nigropria and a new species of Aneuropria Kieffer (Diapriidae: Proctotrupoidea: Hymenoptera) from India. Entomon 25: 193-200.

Rajmohana K, Narendran TC (2000b) Two new genera of Diapriidae (Proctotrupoidea: Hymenoptera) from India. Uttar Pradesh Journal of Zoology 20(1): 21-28.

Rajmohana K, Poorani J, Shweta M, Malathi C (2013) A pictorial guide to Diapriinae genera of India [Online]. Zoological Survey of India \& National Bureau of Agricultural Insect Resources, Calicut, Kerala. http://www.nbair.res.in/Diapriinae/index.php [accessed 3 Oct. 2015] 